ARTICLE

https://doi.org/10.1038/s41467-019-10428-1

\title{
Factors other than hTau overexpression that contribute to tauopathy-like phenotype in rTg4510 mice
}

Julia Gamache (1) ${ }^{1}$, Kellie Benzow ${ }^{2}$, Colleen Forster ${ }^{3}$, Lisa Kemper ${ }^{1}$, Chris Hlynialuk ${ }^{1}$, Eva Furrow (iD ${ }^{4}$, Karen H. Ashe ${ }^{1} \&$ Michael D. Koob (1) ${ }^{2}$

The tauopathy-like phenotype observed in the $\mathrm{rg} 4510$ mouse line, in which human taup301L expression specifically within the forebrain can be temporally controlled, has largely been attributed to high overexpression of mutant human tau in the forebrain region. Unexpectedly, we found that in a different mouse line with a targeted-insertion of the same transgene driven by the same tetracycline-TransActivator (tTA) allele, but with even higher overexpression of tauP301L than rTg4510, atrophy and tau histopathology are delayed, and a different behavioral profile is observed. This suggests that it is not overexpression of mutant human tau alone that contributes to the phenotype in $\mathrm{rg} 4510$ mice. Furthermore we show that the tauopathy-like phenotype seen in $\mathrm{r} \operatorname{Tg} 4510$ requires a 70 -copy tau-transgene insertion in a $244 \mathrm{~kb}$ deletion in Fgf14, a 7-copy tTA-transgene insertion in a $508 \mathrm{~kb}$ deletion that disrupts another five genes, in addition to high transgene overexpression. We propose that these additional effects need to be accounted for in any studies using rTg4510.

\footnotetext{
${ }^{1}$ Department of Neurology, N. Bud Grossman Center for Memory Research and Care, Minneapolis, MN, USA. ${ }^{2}$ Department of Laboratory Medicine and Pathology, Minneapolis, MN, USA. ${ }^{3}$ BLS Histology and IHC Laboratory, Minneapolis, MN, USA. ${ }^{4}$ Veterinary Clinical Sciences (College of Veterinary Medicine), University of Minnesota, Minneapolis, MN 55455, USA. Correspondence and requests for materials should be addressed to K.H.A. (email: hsiao005@umn.edu) or to M.D.K. (email: koobx001@umn.edu)
} 


\section{T} he widely used rTg4510 mouse model of tauopathy recapitulates key features of these human diseases, including progressive age-related neurofibrillary tangles (NFTs), memory impairment and a dramatic loss of neurons in young mice $^{1,2}$. The rapid onset and severity of neuron loss in this model was particularly surprising when first reported given that earlier mouse models incorporating similar human microtubuleassociated protein tau (MAPT) transgenes with the pathogenic P301L mutation do not develop overt atrophy ${ }^{3,4}$. Unlike these other $\operatorname{tau}_{\mathrm{P} 301 \mathrm{~L}}$ overexpression models, however, $\mathrm{rTg} 4510$ uses a responder-driver system to activate transgene expression specifically in forebrain neurons. Responder Tg4510 mice are crossed to a driver line $\mathrm{s}^{5}$ that harbors a tetracycline transactivator (tTA) transgene to generate bi-transgenic rTg4510 (" $r$ " refers to regulatable) progeny. The particularly high level of tau $\mathrm{P}_{\mathrm{P} 301 \mathrm{~L}}$ overexpression specifically in the forebrain of these mice has generally been accepted as the direct cause of the premature gross forebrain atrophy and other tauopathy-like phenotypes in this line, a hypothesis supported by the fact that suppression of $\operatorname{tau}_{\mathrm{P} 301 \mathrm{~L}}$ expression by doxycycline (DOX) halted neuronal loss and improved memory function ${ }^{2}$. Mice from a control line in which wild-type (WT) human tau is expressed at levels roughly equivalent to tau $\mathrm{P}_{\mathrm{P} 301 \mathrm{~L}}$ in $\mathrm{rTg} 4510$ mice do not develop either progressive memory deficits or overt atrophy, supporting the idea that the $\operatorname{tau}_{\mathrm{P} 301 \mathrm{~L}}$ mutant form of tau in particular was the direct cause of these phenotypes in $\operatorname{rg} 4510^{6}$.

Our overall goal when we began the project described here was to determine the precise molecular features of the tau $\mathrm{P}_{\mathrm{P} 301 \mathrm{~L}}$ protein that cause the rapid neurodegeneration phenotype characteristic of $\mathrm{rTg} 4510$, but we realized that this line was an inadequate starting point for this work. Although the transgene construct used to generate $\operatorname{Tg} 4510$ could be systematically altered and used to make new lines by pronuclear injection, the random integration process through which $\mathrm{Tg} 4510$ was established could never be precisely repeated, and any transgenic lines generated from these modified constructs would not adequately match the original $\operatorname{Tg} 4510$ line. We therefore first sought to establish a targeted-insertion equivalent to $\operatorname{Tg} 4510$ to which subsequent targeted-insertion lines expressing specific human tau variants could be precisely matched (an overview of the mouse lines generated and used is shown in Supplementary Fig. 1).

Although we find that mice from this targeted-insertion line (designated rT2/T2) overexpress even more tau $\mathrm{P}_{301 \mathrm{~L}}$ than rTg4510, these mice exhibit significantly delayed overt atrophy and tau histopathology and an abnormal age-related flight response. Using whole-genome sequence analyses, we find that the transgene insertion/deletion (TgINDEL) mutation linked to the confounding effects in $\mathrm{rTg} 4510$ is an approximately 70-copy tau-transgene insertion in a $\sim 250 \mathrm{~kb}$ deletion of the first exons and promoter regions of fbroblast growth factor 14 (Fgf14). Furthermore, we find that the tTA Tg-INDEL allele, which drives tau $_{\mathrm{P} 301 \mathrm{~L}}$ expression in both lines and is known to be sufficient to cause a more limited but progressive neuron loss, is an approximately 7-copy tTA-transgene insertion in a $\sim 500 \mathrm{~kb}$ deletion that disrupts another five annotated genes (Vipr2Ptprn2). Finally, we find that, in the absence of the Fgf14 tauTgINDEL, matching the high level of transgene overexpression in rTg4510 appears to be necessary to cause premature ( $\leq 7$ months) tau histopathology, late-stage ( $>12$ months) overt atrophy, and behavior abnormalities.

\section{Results}

TAU $_{\text {P301L }}$ overexpression and gross forebrain atrophy. We used Flp/Frt recombination to target a single copy of the same tau $\mathrm{P}_{\mathrm{P} 301 \mathrm{~L}}$ transgene used to generate Tg4510 into mouse embryonic stem cells at an intergenic site downstream of collagen type I alpha I (Col1A1), a site previously demonstrated to promote transgene expression without dysregulating endogenous genes ${ }^{7}$. Mice with this single targeted MAPT cDNA transgene insertion are designated T2. In order to match the expression pattern in $\mathrm{rTg} 4510$ mice, these new T2 mice are crossed to the same tTA-driver line ${ }^{5}$ used to generate rTg4510 mice, resulting in rT2 mice. The rT2 mice are again crossed to $\mathrm{T} 2$ mice to generate mice homozygous for the tau $\mathrm{P}_{301 \mathrm{~L}}$ transgene (i.e., rT2/T2, as shown in Supplementary Fig. 1).

We find that although rT2/T2 mice express the same levels of $\operatorname{tau}_{\mathrm{P} 301 \mathrm{~L}}$ mRNA and even higher levels of protein in their forebrains than rTg4510 mice (Fig. 1a, b), rT2/T2 mice do not exhibit the dramatic premature loss of brain mass shown by rTg4510, which lose $20 \%$ of their forebrain mass by 7 months of age (Fig. 1c, d). Gross forebrain atrophy, evident in rTg4510, is also absent in rT2/T2 at 7 months of age (Fig. 1e).

Our experimental design for the work described here included evaluating forebrain mass loss through 7 months, at which point rTg4510 mice exhibit severe forebrain mass loss and obvious gross forebrain atrophy (Fig. 1). Although the rT2/T2 mice do not share these phenotypes by $7 \mathrm{M}$, we had sufficient animal numbers to continue these evaluations at 9 months, and then with fewer animals per time point at 10,13, and 15 months (Fig. 2). The few rT2/T2 animals examined at 13 and 15 months in this pilot experiment each had severe forebrain mass loss and obvious gross forebrain atrophy that matched that of the few remaining background strain-matched rTg4510 examined at similar ages. We used rT2 mice as controls that incorporated the effects of the tTA TgINDEL but with more moderate overexpression of $\mathrm{tau}_{\mathrm{P} 301 \mathrm{~L}}$. We did not find severe forebrain mass loss or obvious gross forebrain atrophy by even 18 months in the rT2 mice, although brain mass may be trending lower in the oldest rT2 mice evaluated. Expected rTg4510 brain mass values based on accumulated data from the Ashe lab are shown for comparison (Fig. 2b).

TAU $_{\text {P301L }}$-TgINDEL in Tg4510 disrupts Fgf14. To determine if an insertion mutation caused by the tau $\mathrm{P}_{\mathrm{P} 301 \mathrm{~L}}$ transgene recombination into the Tg4510 mouse genome could explain the phenotypic difference between $\mathrm{rTg} 4510$ and $\mathrm{rT} 2 / \mathrm{T} 2$, we performed whole-genome sequencing on DNA from a Tg4510 responder mouse using the Illumina HiSeq2500 High-Output system. Assembly and analyses of these reads revealed that approximately 70 head-to-tail copies of the tau transgene form an array that replaces a 243,608 bp region of fibroblast growth factor 14 (Fgf14), the most telomeric gene on the long arm of chromosome 14 (Fig. 3b). Within the insertion array the transgene orientation switches from a $3^{\prime} \rightarrow 5^{\prime}$ orientation to a $5^{\prime} \rightarrow 3^{\prime}$ orientation, however, we are unable to determine precisely at which point in the array this switch occurs (Fig. 3b). We confirmed the transgene insertions sites by polymerase chain reaction (PCR) amplifying and resequencing key junctions and by performing fluorescence in situ hybridization (FISH) on Tg4510 genomic DNA (Supplementary Fig. 2c). The compiled tau transgene monomer sequence and all junction sequences have been submitted to GenBank.

Fgf14 expression is dysregulated in rTg4510 mice. Although transcription of FGF14 has been reported to initiate at over unique 100 start sites $^{8}$, at the time we began our analyses four representative splice variants of Fgfl4 were present in GenBank, and we restricted our analyses to these variants: V1 (NM_010201.4, encodes isoform 1a), V2 (NM_207667.3, encodes isoform 1b), X1 (XM_011244952.1), and X2 (XM_006518549.2). The deletion in $\operatorname{Tg} 4510$ removes the first $219 \mathrm{~kb}$ of $\mathrm{V} 2$ and 

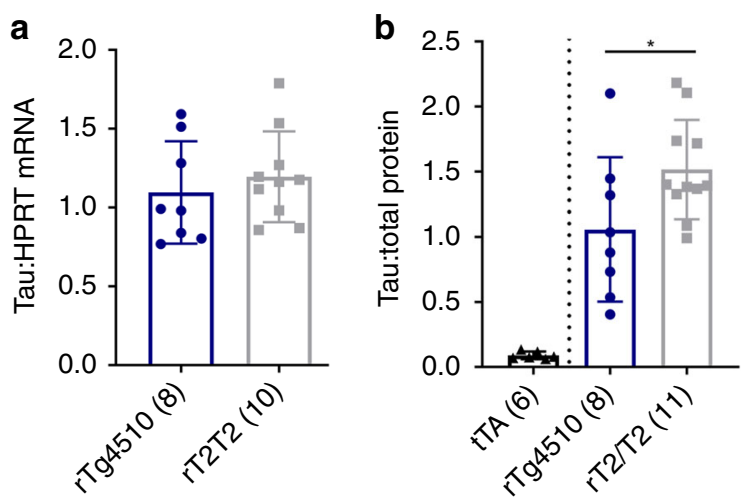

C

d

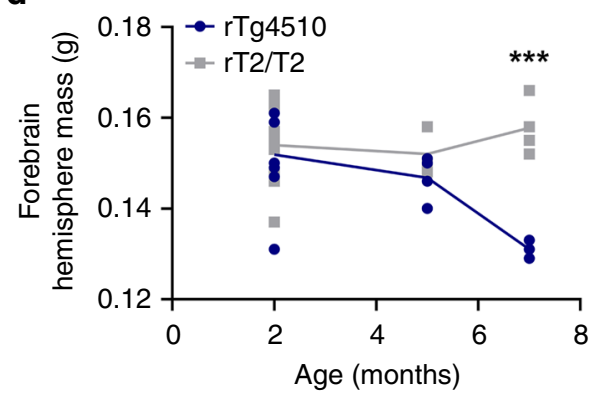

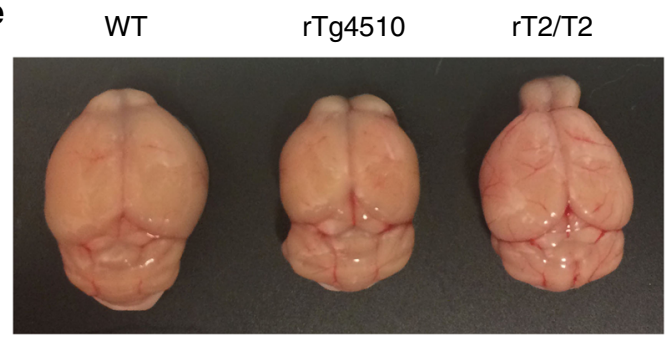

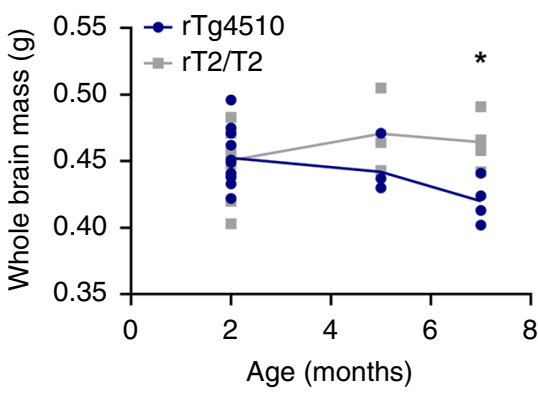

Age (months)

Fig. 1 No premature gross forebrain atrophy in rT2/T2 despite greater overexpression of taup301L. a We used relative qRT-PCR on RNA extracted from mouse forebrain-hemispheres to determine tau expression levels relative to Hprt ( $n$ 's in parentheses). Samples were run in triplicate and the experiment was replicated twice. All data are normalized to a positive calibrator run with each plate to control for inter-plate variability within experiments. An unpaired two-tailed $t$ test was conducted ( $p=0.501, t=0.6882, \mathrm{~d} f=16,95 \% \mathrm{Cl}$ [ -0.21 to 0.41$]$ ). b Protein from mouse forebrains was treated with phosphatase and analyzed by semiquantitative western blot relative to total protein loaded ( $n$ 's in parentheses). A pan-tau antibody (Tau46) was used to quantify fold-overexpression of human tau relative to endogenous tau in tTA mice, and was found to be 17-fold in rT2/T2 and 12-fold in rTg4510. An unpaired two-tailed $t$ test revealed higher overexpression in $\mathrm{rT} 2 / \mathrm{T} 2$ than $\mathrm{rTg} 4510$ ( $p=0.046, t=2.16, \mathrm{~d} f=17,95 \% \mathrm{Cl}[0.010-0.91]$ ). The full blots are shown in Supplementary Fig. 13. c Whole brains were dissected from 2-month (rTg4510 $n=10, S D=0.022 ; r T 2 / T 2 n=11, S D=0.023), 5$-month $(r T g 4510 n=4, S D=0.020 ; r T 2 / T 2 n=3, S D=0.032)$, and 7-month-old mice $(r T g 4510 n=4, S D=0.017 ; r T 2 / T 2 n=4, S D=0.020)$ and weighed. Bonferroni-corrected unpaired two-tailed $t$ tests were conducted for 2 -month $(p=2.56, t=0.1894, \mathrm{~d} f=19,95 \% \mathrm{Cl}[-0.02$ to 0.02$]), 5$-month $(p=0.58$, $t=1.497, \mathrm{~d} f=5,95 \% \mathrm{Cl}[-0.08$ to 0.02$])$, and 7-month ( $p=0.046, t=3.359, \mathrm{~d} f=6,95 \% \mathrm{Cl}[-0.08$ to -0.01$])$ groups. d Forebrain-hemispheres were dissected from 2-month ( $r T g 4510 n=9, S D=0.010 ; r T 2 / T 2 n=10, S D=0.009), 5$-month $(r T g 4510 n=4, S D=0.005 ; r T 2 / T 2 n=3, S D=0.005)$, and 7-month ( $r T g 4510 n=3, S D=0.002 ; r T 2 / T 2 n=4, S D=0.006)$ old mice and weighed. A one-way ANOVA was conducted $(F(5,27)=5.034$, $p=0.0022)$ with Bonferroni's multiple comparisons test for 2 -month ( $p>0.99, t=0.31, \mathrm{~d} f=27,95 \% \mathrm{Cl}[-0.008$ to 0.010$]), 5$-month $(p>0.99, t=0.87$, $\mathrm{d} f=27,95 \% \mathrm{Cl}[-0.010$ to 0.021$])$, and 7-month ( $p=0.0004, t=4.42, \mathrm{~d} f=27,95 \% \mathrm{Cl}[0.011-0.0422]$ ) groups. e Frozen brains from a 7-month female wild-type (left), rTg4510 (middle), and rT2/T2 (right) mouse were thawed and immersion-fixed in 10\% formalin. Data in (a) and (b) are represented as group mean \pm standard deviation. Data in $(\mathbf{c})$ and $(\mathbf{d})$ are represented as connected means. ${ }^{\star} p \leq 0.05,{ }^{\star \star \star} p \leq 0.001$. Source data are provided as a Source Data file

terminates $266 \mathrm{~kb}$ upstream of the transcription start site for V1. Overall, this removes the promoters and first exons of variants $\mathrm{V} 2$, X1, and $\mathrm{X} 2$, leaving the coding region of only variant $\mathrm{V} 1$ intact (Fig. 3b). Available antibodies to Fgf14 protein do not distinguish between the products of these splice variants, and as a result Western blot analyses of Fgf14 differences between these lines was uninformative with respect to altered ratios of Fgf14 isoforms. We performed quantitative real-time PCR (qRT-PCR) of splice variants using RNA extracted from forebrain tissue of rTg4510, Tg4510, and nontransgenic (NT) mice and found that rTg4510 mice express 5.6-fold higher mRNA levels of variant V1 than NT mice and 2.8-fold higher levels of V1 than Tg4510 mice in their forebrains (Fig. 4a). Both rTg4510 and Tg4510 mice exhibit decreased expression of Fgf14 variants V2, X1, and X2, presumably due to haploinsufficiency (Fig. $4 \mathrm{~b}-\mathrm{d}$ ).

tTA-TgINDEL in rTg4510 and rT2/T2 disrupts five more genes. We also performed whole-genome sequencing on DNA from an rT2/T2 mouse. We confirmed the expected configuration of the targeted tau $\mathrm{P}_{\mathrm{P} 01 \mathrm{~L}}$ transgene insertion in this line and also defined the tTA-TgINDEL mutation used to drive expression both in this line and in $\mathrm{rTg} 4510$. We found that approximately 7 head-to-tail copies of the tTA transgene form an array that replaces $508,119 \mathrm{bp}$ in a gene-rich region of mouse chromosome 12 near the immunoglobulin heavy chain complex (Fig. 5). Similar to the configuration within the Fgf14 tau-TgINDEL allele, the transgene orientation within the tTA insertion array switches from a $3^{\prime} \rightarrow 5^{\prime}$ orientation to a $5^{\prime} \rightarrow 3^{\prime}$ orientation, although in this instance a cloning vector DNA fragment is also incorporated at this inversion point. The massive genomic deletion in the tTATgINDEL removes the $3^{\prime}$ portion of Vasoactive intestinal peptide receptor 2 (Vipr2), the $5^{\prime}$ portion of protein tyrosine phosphatase, receptor type, $N$ polypeptide 2 (Ptprn2), and encompasses all of the annotated genes WD repeat domain 60 (Wdr60), extended synaptotagmin-like protein 2 (Esyt2), and non-SMC condensin II complex, subunit G2 (Ncapg2), as well as several predicted but uncharacterized genes (e.g., Gm20658). We confirmed the transgene insertions sites by PCR amplifying and resequencing key junctions (Supplementary Fig. 3). All junction sequences and 


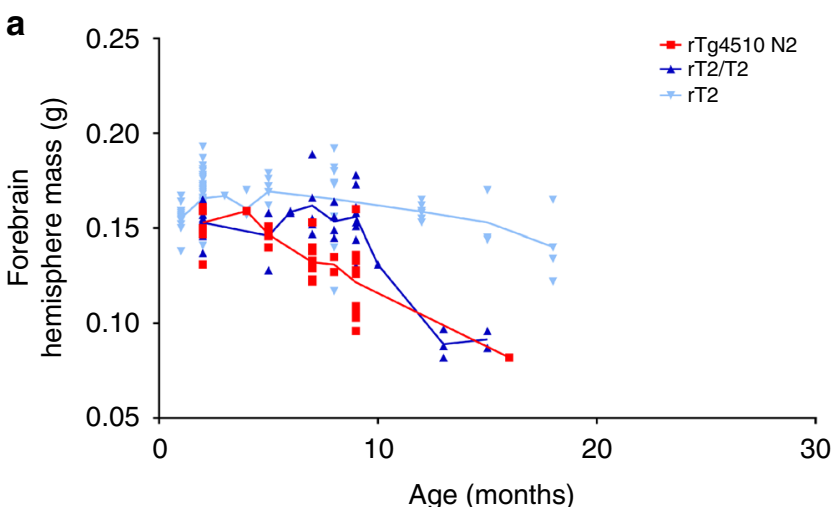

b

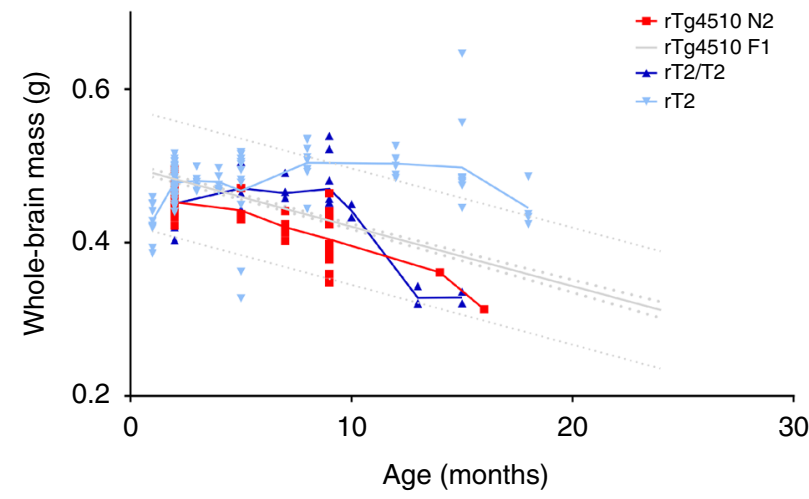

Fig. 2 Pilot experiments with aged $\mathrm{rT} 2$ and $\mathrm{rT} 2 / \mathrm{T} 2$. Forebrain-hemisphere $\mathbf{a}$ and whole brain $\mathbf{b}$ mass were measured for rT2/T2 $(n=32), \mathrm{rT} 2(n=$ 120), rTg4510 F1 (FVB/129S6) ( $n=554$, accumulated data from Ashe lab), and $r$ Tg4510 N2 (FVB $\times$ FVB/129S6) $(n=32)$. Tissue samples were collected at varying ages, with limited numbers at the oldest ages (>12 months). Note that the genetic background of rTg4510 N2 is matched to $\mathrm{rT2} / \mathrm{T} 2$ and that of $\mathrm{rTg} 4510 \mathrm{~F} 1$ is matched to $\mathrm{rT} 2$. A comparison between rT2 and rT2/T2 reveals that severe loss in forebrain mass similar to rTg4510 is only observed in mice $>12$ months and only at the higher levels of transgene overexpression. Lines represent connected means. Thick dotted lines for rTg4510 F1 data represent 95\% confidence bands while thin dotted lines represent $95 \%$ prediction bands. Source data are provided as a Source Data file

the compiled tTA transgene monomer sequence, which includes a $\mathrm{Ca}^{2+}$-calmodulin kinase II (CaMKII) promoter fragment, have been submitted to GenBank.

Transgene expression and Fgf14-TgINDEL accelerate pathology. We evaluated human tau histopathology in tissue taken from the somatomotor cortex and hippocampal CA1 of 2-, 5-, and 7month-old male and female rTg4510, rT2/T2, and rT2 mice using MC1, AT8, CP13, and PHF1 antibodies as well as a Bielschowsky (Biels.) silver stain. Semiquantitative analyses of stained sections (Supplementary Figs. 4-8) revealed similar pathological forms of tau in the first two of these three lines, although these accumulated at earlier ages and to a greater extent in $\mathrm{rTg} 4510$ than in rT2/T2 (Fig. 6b). Western blot densitometry of phosphorylated tau (pTau) showed higher signal in transgene-positive and transgene-negative control mice, but not between rTg4510 and rT2/T2, possibly due to variability in the data (Supplementary Figs. 9 and 10). However, analyses of insoluble high-molecularweight tau was more consistent with the histopathology data (Supplementary Fig. 11). When we examined tissue samples from the $\mathrm{rT} 2$ line with less extreme tau $\mathrm{P}_{\mathrm{P} 01 \mathrm{~L}}$ overexpression $(8.5 \times$ endogenous mouse tau level in rT2 vs. $17 \times$ in $\mathrm{rT} 2 / \mathrm{T} 2$, see
Supplementary Fig. 12) we found only limited instances of pathological-associated forms of human tau protein in a few tissue samples from 7-month-old mice (Fig. 6b).

Age-related behavior changes in rT2/T2. The Morris Water Maze has been regularly used to detect memory impairment in rTg4510 mice ${ }^{1,2}$, but 7-month-old rT2/T2 were too hyperactive in our hands to evaluate them in this task (see Fig. 7). We have not observed this high intensity flight response phenotype in mice in our rTg4510 colony. Nest building activity, which is a natural behavior in mice that has been used as a measure of behavior dysfunction in other mouse models of tauopathy ${ }^{9}$, was severely impaired in the rT2/T2 mice by 8.5 months whereas the rT2 mice exhibited normal nest building activity (Fig. 7a, b). Additional evaluation of the behavior phenotype in 8.5-month-old rT2/T2 mice using open field analysis suggest that these mice are hyperactive as indicated by distance traveled (Fig. 7c), but do not spend more time moving (Fig. 7d) and do not exhibit a higher level of anxiety (Fig. 7e). As these mice age (12.5 months), distance traveled is reduced, but apparently primarily due to significant reductions in time moving (Fig. $7 c, d$ ). We did not examine nest building activity or open field behavior in agematched rTg4510 mice.

\section{Discussion}

Of more than 300 therapeutic agents that had been reported by 2011 to effectively treat deficits in transgenic mouse models of Alzheimer's disease, none had shown significant clinical benefit in human clinical trials ${ }^{10}$. An expert advisory panel of academic, industry, and government scientists convened to address this failure recommended in its best practices report "choosing models for preclinical studies that exhibit significant and wellcharacterized pathology relevant to the disease process of interest (that is, amyloid plaques, tau pathology, neuronal loss, oxidative stress/inflammatory changes, and so on)."10 Perhaps due in part to this recommendation, the rTg4510 mouse model of tauopathy, which exhibits profound premature neurodegeneration and NFTs, has become widely used for both basic and preclinical studies. Among models of tauopathy, rTg4510 mice display the most dramatic loss of neurons, and this loss progresses rapidly in young animals (2-7 months) (Fig. 1). However, we have now found that overexpression of the pathogenic tau variant in these mice is not sufficient to cause this premature and robust agerelated loss of forebrain mass and gross forebrain atrophy, as originally and currently thought.

Two distinct mutant alleles are combined in rTg4510 mice: (1) the $\operatorname{tau}_{\mathrm{P} 301 \mathrm{~L}}$-TgINDEL and (2) the tTA-TgINDEL needed to drive expression from the tau $\mathrm{P}_{\mathrm{P} 301 \mathrm{~L}}$ transgene allele. Both of these mutations were generated by pronuclear injection of synthetic mini-gene constructs into mouse single-cell embryos and were selected from among the many random integration events that occurred by screening for alleles that conferred the desired phenotype. In neither case, however, were the selected mutations themselves mapped or characterized. Our work presented here together with growing evidence from other groups indicates that the attractive $\mathrm{rTg} 4510$ tauopathy phenocopy found by screening for pathology similar to those found in human tauopathies arises from unintended dysfunctions conferred by the tau $\mathrm{P}_{\mathrm{P} 301 \mathrm{~L}}$ and $\mathrm{tTA}$ TgINDEL mutations.

Precisely defining the effects of the Vipr2-Ptprn2 tTATgINDEL allele on the tauopathy phenocopy of rTg4510 is complex and in some ways problematic. The pronuclear injection experiment used to make this tTA allele, which is nominally expressed from a CamKII promoter fragment, generated lines with a wide array of expression patterns ${ }^{5}$ and the primary 

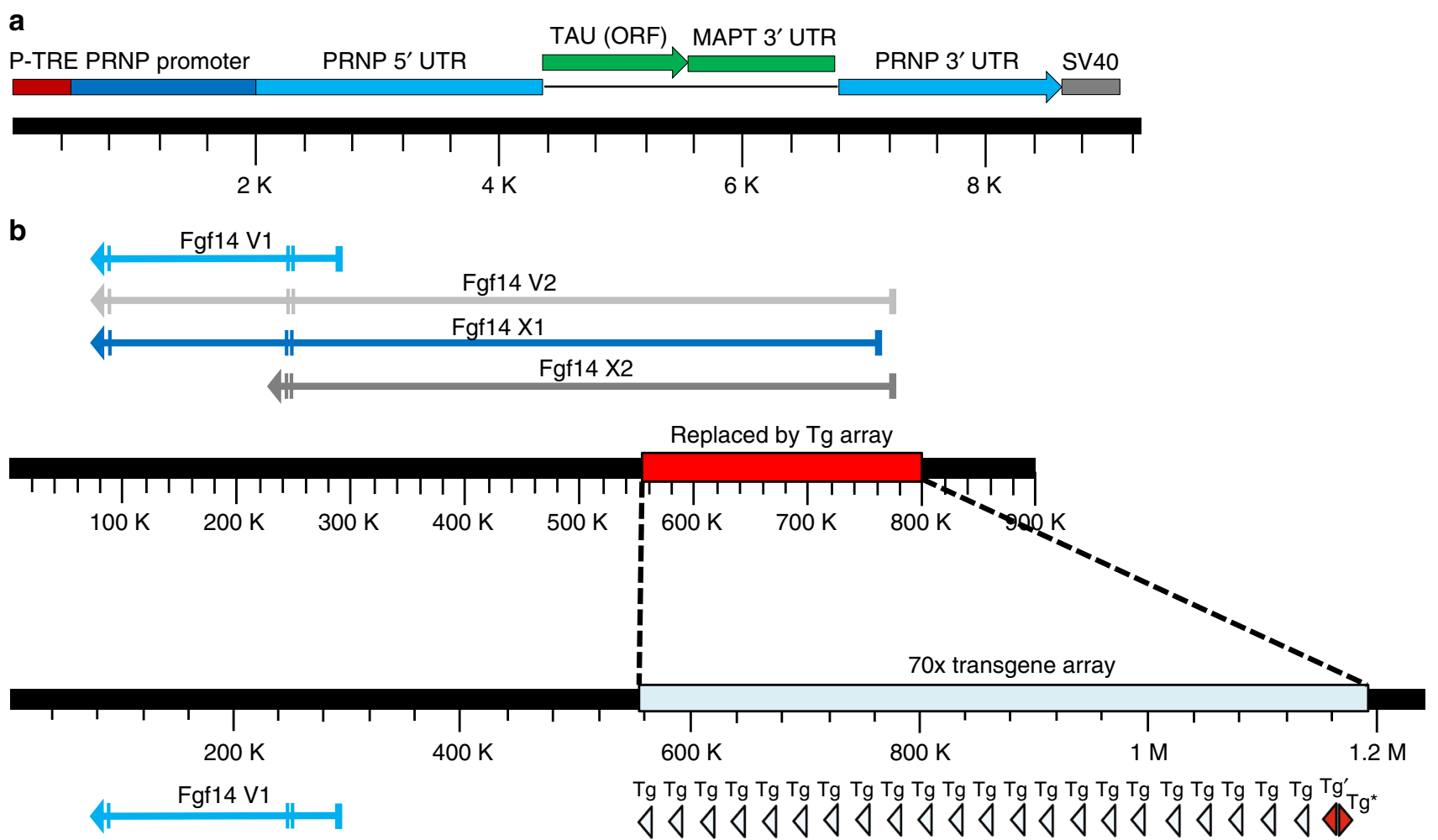

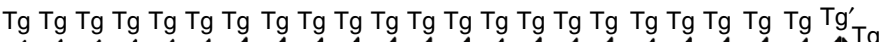
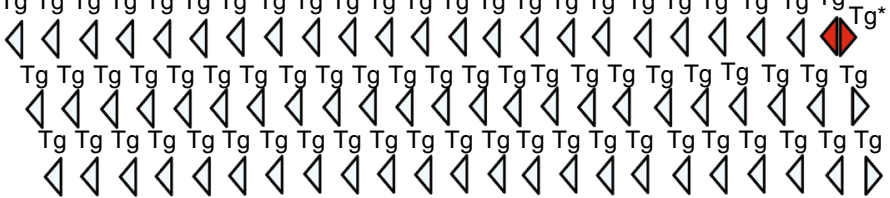

Fig. 3 Fgf14 is disrupted by a tau transgene array in Tg4510 mice. a Structure of the tau transgene monomer including the tetracycline response element (TRE) promoter, prion protein gene (Prnp) sequences, tau cDNA open reading frame (ORF), Mapt 3' untranslated region (UTR), and SV40 polyadenylation signal. b Diagram of Fgf14 mRNA splice variants and disruption by the transgene array. Vertical hashmarks in splice variants represent exons while arrows indicated the direction of synthesis. The red rectangle on the non-transgenic allele (top) represents the 243,608 bp deletion while the light blue rectangle on the transgenic allele (bottom) represents the approximately 70-copy insertion of the Tg multimer array. Tg, transgenes are light blue triangles, $\mathrm{Tg}^{\prime}$, partial transgene copy red triangle in the $3^{\prime} \rightarrow 5^{\prime}$ orientation, $\mathrm{Tg}^{\star}$, partial transgene copy red triangle in the $5^{\prime} \rightarrow 3^{\prime}$ orientation

phenotype originally used to select this particular tTA-TgINDEL allele was functional tTA-driven overexpression selectively in mouse forebrain neurons ${ }^{5}$. Since genomic regulators retained in flanking sequences at each insertion site dictate the unique temporal and spatial expression pattern of tTA from each mutant allele, we were not surprised to find that four of the five annotated genes disrupted by this particular recombination event (Vipr2, Ptprn2, Wdr60, and Esyt2, Fig. 5) have prominent forebrain expression patterns (https://www.ncbi.nlm.nih.gov/gene/) $)^{11}$. We note that loss of both copies of the fifth annotated gene, encoding the chromosome condensin Ncapg2, results in embryonic lethality ${ }^{12}$. If nothing else, the unique tTA expression pattern generated from the combination of the CamKII promoter fragment within the context of the Vipr2-Ptprn2 tTA-TgINDEL mutation defines the $\operatorname{tau}_{\mathrm{P} 301 \mathrm{~L}}$ expression pattern in $\mathrm{rTg} 4510$, and likely defines the tissue specificity of the tauopathy phenocopy as well. Because we did not generate an alternative targeted insertion of a tTA transgene for our current work, we cannot determine if the loss of the genes disrupted by the Vipr2-Ptprn2 tTA-TgINDEL directly affect the tauopathy phenocopy of rTg4510.

Because the tau transgene is expressed from a synthetic tTAresponsive promoter, tTA activity and tau expression are intrinsically linked, and as a result conclusively extricating the impacts of tau P301L $_{\text {f }}$ from those of $\mathrm{tTA}$ activity on phenotypes in $\mathrm{rTg} 4510$ may not be possible. The Vipr2-Ptprn2 tTA-TgINDEL allele alone has been shown to be sufficient to cause a progressive neuron loss that leads to obvious degeneration of the dentate gyrus ${ }^{13}$.
Suppression of tTA activity during the first 6 weeks of postnatal development with Dox has been shown to eliminate this observed neuron loss ${ }^{13}$, presumably by eliminating tTA binding and subsequent unintended activation of non-target gene expression at undefined loci in the genome. We note that the $490+$ cognate tTA binding sites within the Fgfl4 tau-TgINDEL would also be expected to modify binding of excess tTA to and transcription activation at noncognate genomic sites, potentially complicating the interpretation of direct comparisons between rTg4510 mice and tTA-only littermates. For example, neuron loss in rTg4510 mice is less obvious in the dentate gyrus than in other regions of the hippocampus and cortex ${ }^{1}$, suggesting that the course of the documented tTA-driven neuronal toxicity and neuron loss is significantly altered in $\mathrm{rTg} 4510$ mice and may not contribute significantly to neuron loss. The design of the $\mathrm{rTg} 4510$ model precludes testing the alternative but not mutually exclusive hypotheses that overexpression of $\operatorname{tau}_{\mathrm{P} 301 \mathrm{~L}}$ or $\mathrm{tTA}$ in this early developmental window is necessary to cause neuron loss. Administering Dox to rTg4510 mice from conception to 6 weeks, followed by removal of Dox resulted in robust tau histopathology by 54 weeks of age; neuron loss was reported to be absent, but data for neuron loss was not shown ${ }^{14}$. In addition, Dox suppression during development has been shown to result in a lower level of transgene overexpression in a model in which the Vipr2Ptprn2 tTA-TgINDEL allele drives expression of a CamKII target transgene ${ }^{15}$. If this is also true for expression from the Fgflit tauTgINDEL, then the post-suppression level of transgene 
a

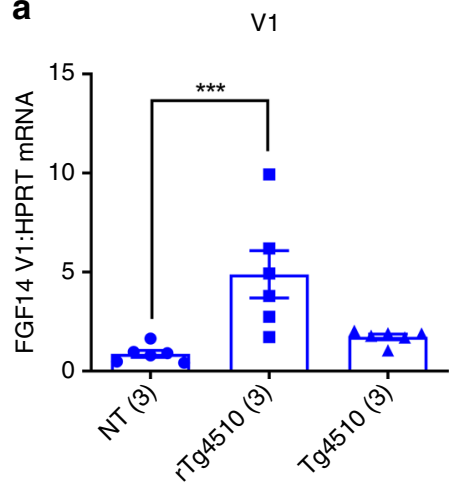

C

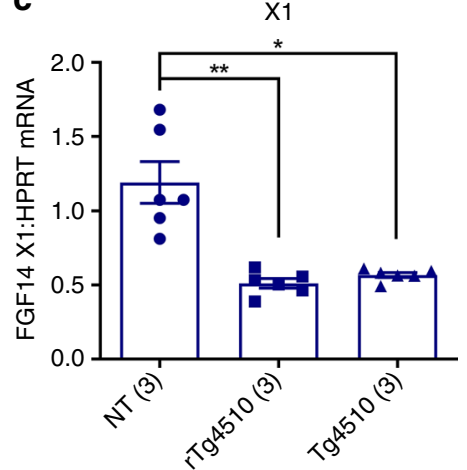

b

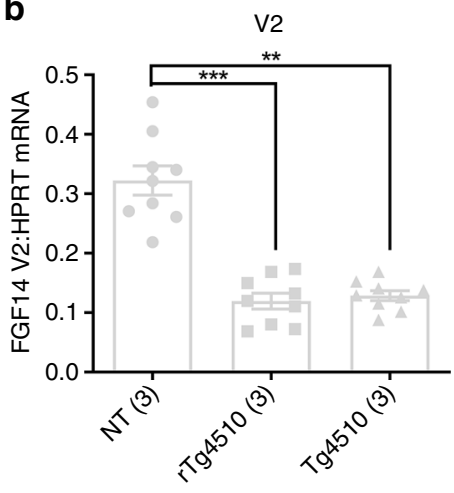

d

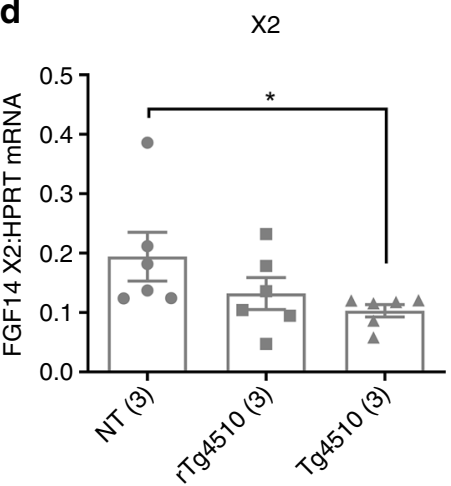

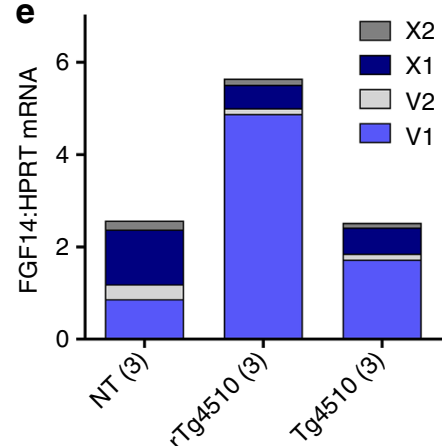

Fig. 4 Dysregulation of Fgf14 mRNA splice variants in rTg4510 and Tg4510 mice. Expression levels in the rostral one-third of dissected forebrainhemispheres of Fgf14 variants V1 (a), V2 (b), X1 (c), and X2 (d) in nontransgenic (NT), rTg4510, and Tg4510 mice (n's in parentheses). Expression levels of each variant were determined by qRT-PCR relative to housekeeping gene Hprt. All samples were run in duplicate and data points from both experiments are shown. Kruskal-Wallis tests were performed for $\mathrm{V} 1(H=13.2, \mathrm{~d} f=2, p<0.0001), \mathrm{V} 2(H=17.5, \mathrm{~d} f=2, p<0.0002), \mathrm{X} 1(H=12.3, \mathrm{~d} f=2, p=0.0001)$, and $\mathrm{X} 2(H=6.9, \mathrm{~d} f=2, p=0.026)$ groups, followed by Dunn's multiple comparisons tests. All data were normalized to a positive calibrator, and are expressed as the group mean \pm standard error. e Compiled group mean data for all variants. ${ }^{\star} p \leq 0.05,{ }^{\star \star} p \leq 0.01,{ }^{\star \star \star} p \leq 0.001$. Source data are provided as a Source Data file

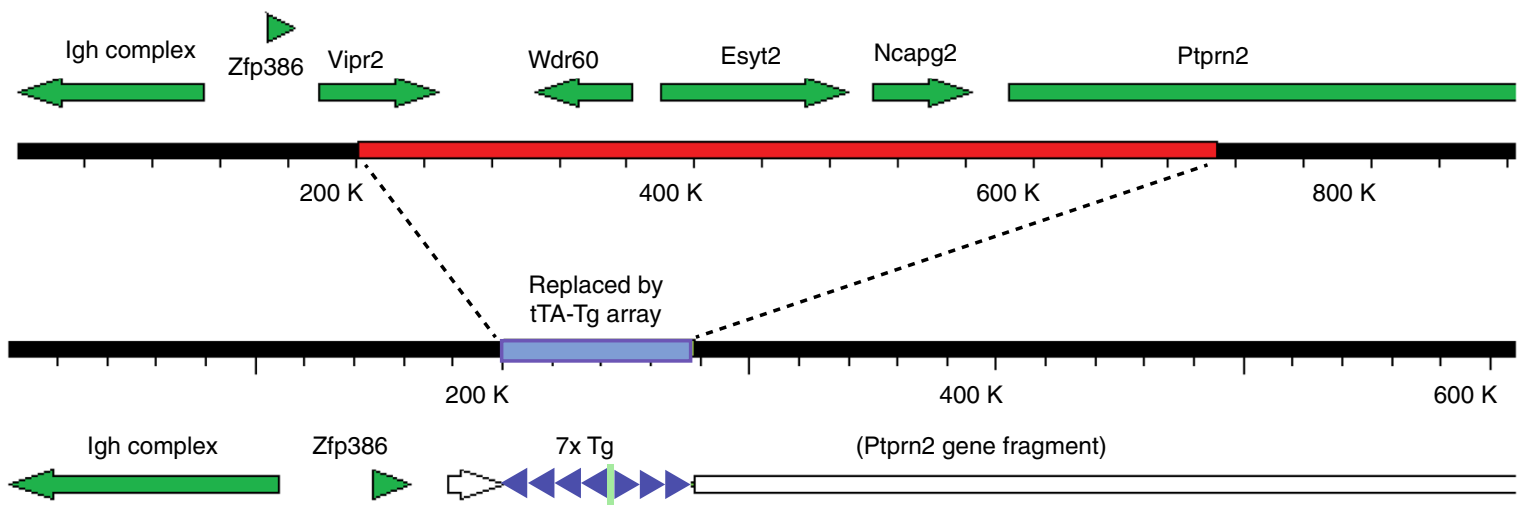

Fig. 5 The tTA transgene array in rTg4510 and rT2/T2 mice disrupts genes on chromosome 12. Diagram of the uninterrupted chromosomal region and its disruption by the tTA-TgINDEL mutation. Tg, transgenes are blue triangles, cloning vector in inversion point of Tg array is a green line, uninterrupted genes are filled arrows, and partially deleted gene fragments are white arrows

overexpression following Dox suppression during development ${ }^{14}$ may not be sufficient to drive neuron loss.

The fact that the Vipr2-Ptprn2 tTA-TgINDEL allele is not genetically linked to the Fgf14 tau-TgINDEL in rTg4510 has allowed us to use this same allele to drive expression in our new targeted-insertion rT2/T2 mice, and thereby matching the pattern of transgene expression and incorporating Vipr2-Ptprn2 tTATgINDEL-linked confounding effects essentially identical to those found in $\mathrm{rTg} 4510$. Our new targeted-insertion $\mathrm{rT} 2 / \mathrm{T} 2$ mice overexpress tau $_{\mathrm{P} 301 \mathrm{~L}}$ at levels even higher than in $\mathrm{rTg} 4510$ mice
(Fig. 1a, b) from the same basic transgene construct (Fig. 3a), but only the rTg4510 mice exhibit robust age-related loss of forebrain mass and gross forebrain atrophy prior to one year of age (Fig. 1c-e). This result clearly demonstrates that a functional contribution from the Fgf14 tau-TgINDEL mutation other than $\operatorname{tau}_{\mathrm{P} 301 \mathrm{~L}}$ overexpression is a necessary cause of this premature overt atrophy. By comparing tau $\mathrm{P}_{\mathrm{P} 301 \mathrm{~L}}$ histopathology in tissue samples from rT2/T2 and rTg4510 mice (Fig. 6, Supplementary Figures 4-11), we are also able to conclude that confounding contributions from the Fgf14 tau P301L -TgINDEL mutation 
a

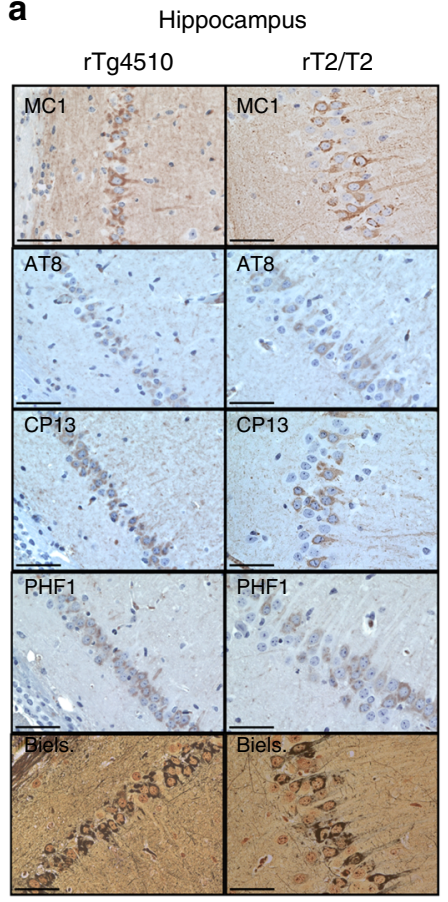

Cortex

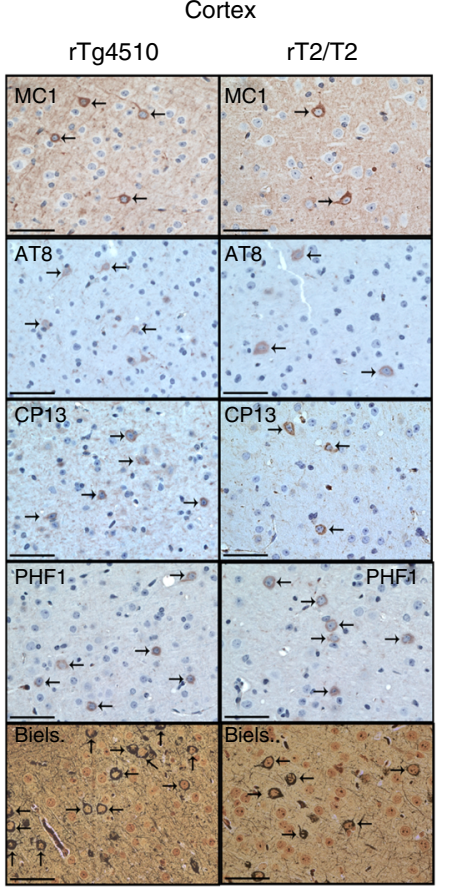

b

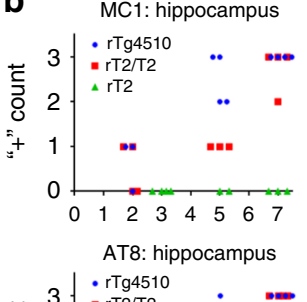

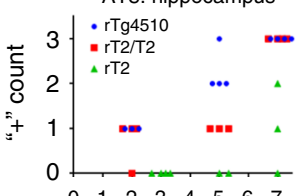

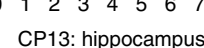

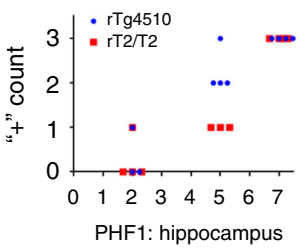

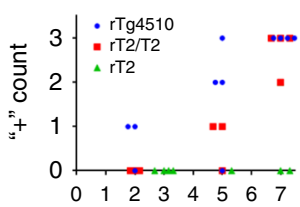

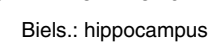

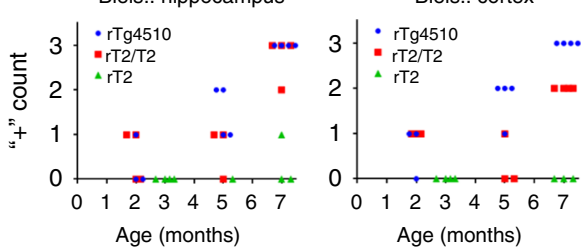

Fig. 6 Comparison of tau histopathology in rT2, rT2/T2, and rTg4510. a Representative 40x images taken from somatomotor cortex and hippocampal CA1 of 7-month-old male rTg4510 and rT2/T2 mice. Stains used to visualize pathological tau aggregates included MC1, AT8, CP13, PHF1, and Bielschowsky (Biels.) silver stains, as indicated. Arrows indicate moderate to prominent positive labeling of tau deposits in cortex, while prominent labeling in hippocampus required no arrows because most or all of the cells exhibited positive labeling. Scale bars represent $50 \mu \mathrm{m}$. b Summary of semiquantitative analysis of tau histopathology progression in rTg4510 ( $n=32 \mathrm{M}, 45 \mathrm{M}, 47 \mathrm{M}), \mathrm{rT2} / \mathrm{T} 2(n=42,35,47 \mathrm{M})$, and rT2 $(n=42,25,37 \mathrm{M})$ mice. Multiple linear regression analyses revealed significant regressions for every stain in both regions: AT8 cortex $\left(F(3,18)=55.7, R^{2}=0.89, p<0.0001\right)$, AT8 hippocampus $\left(F(3,18)=25.8, R^{2}=0.78, p<0.0001\right)$, Biels. cortex $\left(F(3,18)=14.2, R^{2}=0.65, p<0.0001\right)$, Biels. hippocampus $\left(F(3,18)=17.3, R^{2}=0.70, p<0.0001\right)$, $\mathrm{CP13}$ cortex $\left(F(3,18)=26.3, R^{2}=0.78, p<0.0001\right), \mathrm{CP13}$ hippocampus $\left(F(3,18)=40.6, R^{2}=0.85, p<0.0001\right), \mathrm{MC1}$ cortex $\left(F(3,18)=34.2, R^{2}=0.83\right.$, $p<0.0001)$, MC1 hippocampus $\left(F(3,18)=24.0, R^{2}=0.77, p<0.0001\right)$, PHF1 cortex $\left(F(3,18)=18.3, R^{2}=0.71, p<0.0001\right)$, PHF1 hippocampus $(F(3,18)=$ $17.2, R^{2}=0.70, p<0.0001$ ). Also using multiple linear regression, a significant difference between $r \mathrm{Tg} 4510$ and $r \mathrm{~T} 2 / \mathrm{T} 2 \mathrm{y}$-intercepts was found for AT8 in the cortex $(p=0.017)$ and between $r T g 4510$ and rT2/T2 progression of pathology (slope) for Biels. in the cortex $(p=0.024)$. A significant difference between rT2/T2 and rT2 $y$-intercepts was found for AT8 in the cortex $(p=0.014)$ and between rT2/T2 and rT2 progression of pathology in MC1 in the hippocampus $(p=0.0006)$, MC1 in the cortex $(p=0.002)$, AT8 in the cortex $(p<0.001)$, PHF1 in the hippocampus $(p=0.03)$, PHF1 in the cortex $(p=$ 0.009 ), and Biels. in the hippocampus ( $p=0.02)$. A significant difference between rTg4510 and rT2 progression of pathology was found for MC1 in the hippocampus $(p=0.0004)$, MC1 in the cortex $(p=0.001)$, AT8 in the cortex $(p<0.0001)$, PHF1 in the cortex $(p=0.0007)$, Biels. in the hippocampus $(p=0.004)$, and Biels. in the cortex $(p=0.0006)$. Source data are provided as a Source Data file

accelerate the rate at which and the extent to which tau $\mathrm{P}_{\mathrm{P} 01 \mathrm{~L}}$ histopathology develops in rTg4510. Finally, we found that 7month-old rT2/T2 mice in our hands develop an age-related high intensity flight response phenotype that prevented us from testing them in the Morris Water Maze, the task that has been used to measure memory impairment in $\mathrm{rTg} 4510$ mice $^{1,2}$.

We found that the random recombination event that integrated the $\operatorname{tau}_{\mathrm{P} 301 \mathrm{~L}}$ transgene array into the mouse genome in $\mathrm{Tg} 4510$ deleted $244 \mathrm{~kb}$ of the Fgf14 gene (Fig. 3), and that this disruption results in the dysregulation of the ratios of $F g f 14$ splice variants in mice that carry this mutation (Fig. 4). Unfortunately for anyone attempting to determine molecular mechanisms of tau-related dysfunctions using rTg4510, confounding dysfunctions in Fgf14 have the potential to impact almost every aspect of neurobiology, including synaptic transmission, plasticity, and neurogenesis ${ }^{16}$. The splice variants of the internal growth factor Fgf14 differ in their N-terminal amino acid sequences, which contribute to their subcellular localization and their ability to regulate the voltagegated sodium channels with which they interact ${ }^{16-24}$. The fact that unbiased transcriptome analyses of the extent of alternative promoter usage in adult human brains identified FGF14 as one of the few outlier genes with more than 100 transcription start sites strongly suggests that differential regulation of this gene is critical for establishing and maintaining normal brain function ${ }^{8}$. The deletion of $244 \mathrm{~kb}$ of this gene in the Fgf14 tau P $301 \mathrm{~L}_{\text {-TgINDEL }}$ mutation would severely compromise the differential regulation 
a

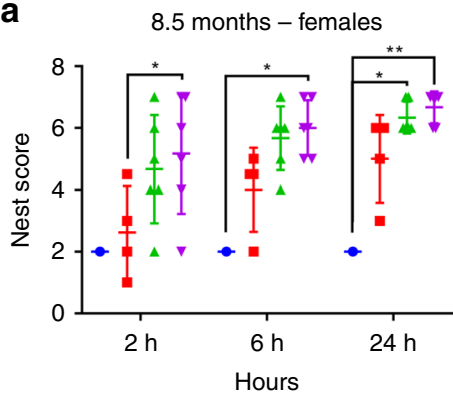

b

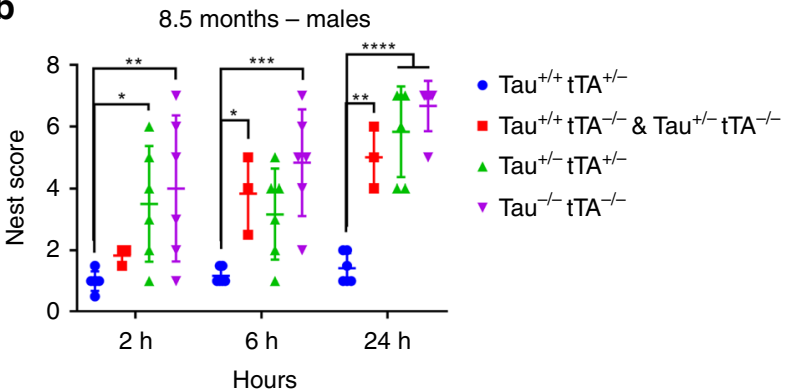

C

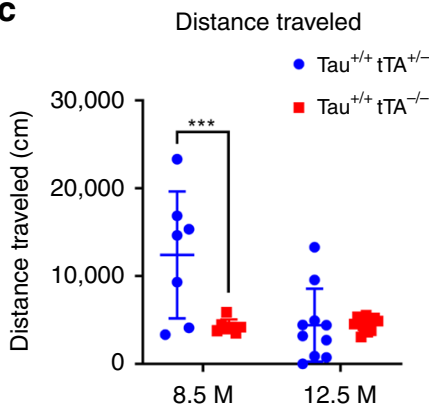

d

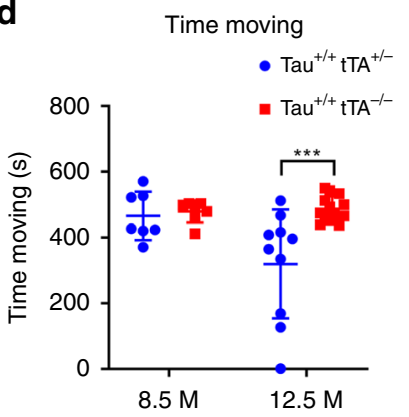

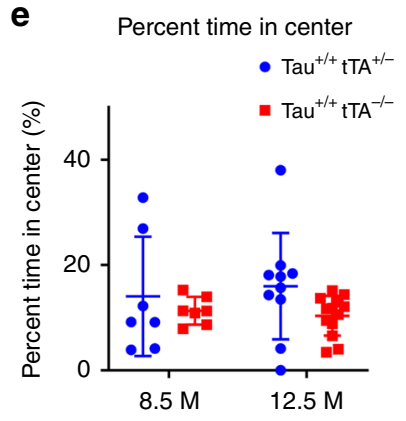

Fig. 7 Nesting and open field behavior in rT2/T2 mice. $\mathbf{a}$, b At 8.5 months of age, rT2/T2 $(n=7), \mathrm{rT2}(n=12)$, and control littermates $\left(\mathrm{Tau}^{+} /+\mathrm{tTA}-/-n=\right.$

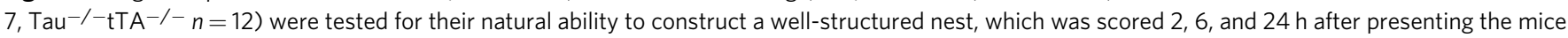
with a fresh nestlet. An overall sex effect was detected indicating that females tended to build better nests than males. Therefore, each sex was analyzed separately. An impairment in nesting was observed in both male and female rT2/T2 mice, with rT2 mice performing significantly better than rT2/T2 mice by $24 \mathrm{~h}$. For females (a), a two-way ANOVA revealed a significant main effect of genotype $(F(3,39)=12.81, p<0.0001)$. For males (b), a two-way ANOVA also revealed a significant main effect of genotype $(F(3,51)=28.18, p<0.0001)$. Tukey's multiple comparisons test were run to compare genotypes within time points. c-e rT2/T2 and control littermates were tested in the open field at 8.5 months of age ( $n=7$ per group) and at 12.5 months of age $\left(\mathrm{Tau}^{+/+} \mathrm{tTA}^{+/-} n=10, \mathrm{Tau}^{-/-} \mathrm{tTA}^{-/-} n=13\right) . \mathrm{rT} 2 / \mathrm{T} 2$ mice exhibit signs of hyperactivity in that they travel significantly farther in the open field than controls at 8.5 months (two-way ANOVA: $F(1,33)=9.395$, main effect of genotype $p=0.0043$ ), however, this declines to normal distance by 12.5 months (c). This shorter distance traveled at 12.5 months is reflected in less time spent moving (two-way ANOVA: $F(1,33)=7.748$, main effect of genotype, $p=0.0088)(\mathbf{d}) . r T 2 / T 2$ mice do not exhibit signs of anxiety, as they spend about the same amount of time in the center of the field as controls (e). Data represent mean \pm standard deviation. ${ }^{\star} p<0.05,{ }^{\star \star} p<0.01,{ }^{\star \star \star} p<0.001,{ }^{\star \star \star \star} p<0.0001$. Source data are provided as a Source Data file

of these functionally distinct $\mathrm{Fg} f 14$ isoforms by removing a large percentage of these transcription start sites and alternative $\mathrm{N}$ terminal coding sequences (Figs. 3 and 4). A translocation within a region of the human FGF14 gene that is nearly identical to the syntenic segment of Fgf14 disrupted by the tau transgene in rTg4510 has been reported to be linked to phenotypes ranging from ataxia (mother) to microcephaly and severe mental retardation (proband) ${ }^{25}$, suggesting that these types of FGF14 disruptions may increase susceptibility to significant loss of brain mass and function.

Comparisons between the hemizygous rT2 line, which overexpresses tau $_{\mathrm{P} 301 \mathrm{~L}}$ at levels over $70 \%$ of that in $\mathrm{rTg} 4510$ mice (Supplementary Fig. 12), and the homozygous rT2/T2 line, with overexpression levels significantly higher than those of rTg4510, has allowed us to determine that high-transgene overexpression is a third critically important contributor to the tauopathy phenocopy in $\mathrm{rTg} 4510$ mice. By comparing tau $\mathrm{P}_{\mathrm{P} 01 \mathrm{~L}}$ histopathology in tissue samples from rT2 to those from rT2/T2 mice (Fig. 6b) we are able to conclude that pushing the level of tau $\mathrm{P}_{\mathrm{P} 01 \mathrm{~L}}$ overexpression to match or exceed that found in $\mathrm{rTg} 4510$ dramatically accelerates the rate and extent to which tau histopathology develops. Open field testing (Fig. 7) enabled us to quantify the hyperactivity phenotype observed in $\mathrm{rT} 2 / \mathrm{T} 2$ mice and our results suggest that this phenotype is linked to the level of tau hyper- or over-expression in these models. These analyses revealed that the rT2/T2 mice traveled a greater distance than controls, but did not move more often, indicating that the true difference was the speed at which these mice moved. A similar increase in distance traveled in open field analysis was also seen in a mouse tauopathy model in which tau $\mathrm{P}_{\mathrm{P} 301 \mathrm{~L}}$ was overexpressed from an adenoassociated virus (AAV) vector, although in that model the increase in distance traveled paralleled a similar increase in time moving ${ }^{26}$. Similar to our findings with rT2/T2, the AAV1$\operatorname{tau}_{\mathrm{P} 301 \mathrm{~L}}$ overexpression model exhibited widespread accumulation of tau histopathology by 6 months without overt loss of neurons ${ }^{26}$. The overt atrophy that we did observe in limited numbers of aged rT2/T2 mice ( $>12$ months), also appears to be a phenotype linked to hyperexpression of transgene, since we did not observe overt atrophy in aged rT2 mice (Fig. 2). We note that the overt atrophy that appears to develop in aged rT2/T2 mice is similar to the ages at which neuron loss is seen in another tauopathy mouse model ${ }^{27}$. Further studies with additional control lines (e.g., rT1 without the P301L mutation in the tau transgene) will be needed to conclude whether these impacts of transgene hyperexpression on phenotype in $\mathrm{rT} 2 / \mathrm{T} 2$ are due specifically to extremely high levels of $\operatorname{tau}_{\mathrm{P} 301 \mathrm{~L}}$ in particular, or to less specific cellular stresses caused by extreme overexpression of an exogenous gene in general. Our finding that high levels of tau $\mathrm{P}_{\mathrm{P} 301 \mathrm{~L}}$ overexpression directly leads to measureable dysfunctions in this protein product parallel findings in which overexpression of amyloid precursor protein (APP) causes additional phenotypes not found in models based on endogenous App expression levels ${ }^{28,29}$. Although massive overexpression of a transgene has been used as a means of inducing dysfunctions in many mouse models and is rationalized as accelerating dysfunctions that also might develop more slowly at physiological levels of expression, 
we need to remain cognizant of the fact that the mechanisms underlying these hyperexpression-induced dysfunctions may be unrelated to those that cause disease in patients.

Our work highlights the critical importance of identifying and using proper control lines in any experiments involving transgenic animal models. The classic controls for confounding contributions from the genomic position effects of randomly integrated transgenes has been to characterize populations of experimental lines (e.g., transgene with a mutation) and of control lines (e.g., transgene without a mutation), and then either continue to work with small populations of these lines ( $\geqq 3$ lines) with phenotypes that are representative of the average phenotypes of these characterized lines, or in most cases, with only single representative lines from each population. In almost every instance, lines with outlier phenotypes will have confounding contributions from their unique TgINDEL mutation. Although it may eventually be possible to reproduce the full range of premature tauopathy-like phenotypes found in $\mathrm{rTg} 4510$ by using targeted genomic modifications to generate control lines that more precisely and reproducibly generate the confounding contributions from the Fgf14-TgINDEL mutation (e.g., similar Fgf 14 dysregulation), this would be a new experimental system, and key findings from $\mathrm{rTg} 4510$ would need to be reproduced in this new system. Given the high cost in time and resources involved in making such a replacement, the onus of pursuing that work would have to fall on any group proposing to continue to use rTg4510 in their research. At this point, the targeted-insertion mouse models we are developing offers a system in which the Fgf14-TgINDEL mutation and the potential confounding contributions from this mutation are eliminated. If crossed to mice that carry the Vipr2-Ptprn2 tTA-TgINDEL, as described here, these mice will still have confounding contributions from this activator allele, but this artificial environment would be matched in all mice in the series, with the only difference between lines being the particular tau variant specifically introduced into the targeted tau transgene. Nonetheless, mechanistic studies using our transgenic models would still need to account for possible contributions from the Vipr2-Ptprn2 tTA-TgINDEL and from nonphysiological levels of transgene overexpression.

For more than a decade rTg 4510 mice have been widely used as a model of human tauopathy but without recognizing the necessary confounding contributions from the Fgf14 tau tano1L $^{-}$ TgINDEL, and with only recent and inconsistent attempts to control for the contributions from the previously undefined Vipr2-Ptprn2 tTA-TgINDEL allele ${ }^{13}$. We are explicitly not asserting that any hypotheses regarding tau pathophysiology generated from these studies using the $\mathrm{rTg} 4510$ model are incorrect. However, we believe that in all of these studies it is critical to account for possible contributions from the confounding effects that we have now defined. In particular, we caution against the use of rTg4510 for testing potential disease therapies, as we now know that a therapeutic agent could significantly improve or even eliminate tauopathy-like phenotypes in rTg4510 by incidentally correcting effects such as the contributions from the Fgf14 tau P301L -TgINDEL mutation (i.e., as in rT2/T2) without modifying the intended drug target.

Although we have restricted our work to characterizing rTg4510, we believe that our findings are indicative of a much broader and deeper problem. Classic pronuclear injection technology generates mice in which a transgene construct is typically arrayed as multimers in unpredictable and irreproducible configurations (see Figs. 3 and 5) inserted in random locations into the mouse genome. Genomic position effects on transgene expression patterns as well as gene disruption and dysregulation caused by these random insert events invariably result in dramatic phenotypic variation between the mouse lines generated by this technology. All too often the mouse line selected for detailed characterization from among these many lines has been the line that exhibits the most "significant... pathology relevant to the disease process of interest" 10 , as was the case with $\mathrm{rTg} 4510$. Although such mouse lines are widely and routinely used, many of the TgINDEL mutations in these lines remain only marginally characterized at the genetic level. As we have shown here, advances in genomic DNA sequencing technology now allow us to fully define the TgINDEL mutations in these lines, enabling us to more accurately identify the confounding effects caused by these mutations and to account for their possible impacts on our work.

\section{Methods}

Animals. We generated ES cells with a Col1A1 "Flp-in" integration cassette by electroporating $25 \mu \mathrm{g}$ of purified Colla-frt-hygro-pA Plasmid (Addgene) into V6.5 Mouse Embryonic stem cells (C57BL/6× 129/sv) (Novus Biologicals NBP1-41162 passage 22). After G418 selection, Clone 15 was found to have integrated properly and had a perfect karyotype. All further FRT mediated targeting is to this ES modified cell line (V6.5Colla\#15). A construct was generated that was essentially identical to the construct used to generate Tg4510, but incorporated a Flp-In promoter cassette (PGK promoter-ATG-FRT), and $6 \mu \mathrm{g}$ this construct and $0.5 \mu \mathrm{g}$ pCAGGS-FlpE (Gene Bridges cat\# A201) was transfected into the V6.5Colla\#15 ES cell line. Hygromycin selection at $140 \mathrm{ug} / \mathrm{mL}$ was added day 2 through day 6 , and Hygro resistant ES clones were picked on day 7. DNA was analyzed for $5^{\prime}$ (5ArmCol1A assay primer + TRE start Rev) and 3' (AMP R Reverse + Hygro Connection) junctions, internal Tau (TAU assay F and R), and multiple integration assay (AMP R Reverse + TRE start R) by PCR. Southern blot was done for multiple integration confirmation using EcoRI digested genomic DNA and a 470 bp probe generated from the AmpR gene (Amp F+Amp R). Clone 6 was positive for all assays and the P301L mutation was verified, and this clone was expanded and karyotyped, and mice were generated by injection into blastocysts. These mice were back-crossed five times to FVB prior to generating the T2/T2 lines. ES Cell Assay Primer sequences: 5ArmCollA pcr assay (5'-CAGGTGCACAGCATTGCGGACAT G-3'), TRE Start Rev (5'-ATTGCTCCAGGCGATCTGAC-3'), Amp R Reverse (5-GGAATAAGGGCGACACGGAA-3'), Hygro Connecton (5'-ATCCACGCCCT CCTACATCGAA-3'). Tau Assay F (5'-GTTCGAAGTGATGGAAGATCACG-3'), and Tau Assay R (5'-TTGGGTGGAGTACGGACCA-3'). PCR Probe Primers sequences: Amp F (5'-CCTCCATCCAGTCTATTAATT-3') and Amp R (5'-TCCT TGAGAGTTTTCGCCCCG-3').

To generate tau-homozygous rT2/T2 mice from tau-hemizygous T2 mice, transgene-activated hemizygous males $(\mathrm{CKTTA}+/-\mathrm{Tau}+/-)$ were bred to nonactivated hemizygous females (CKTTA $-/-\mathrm{Tau}+/-$ ), resulting in tau homozygous progeny $(\mathrm{Tau}+/+)$. To maintain the $\mathrm{rT} 2 / \mathrm{T} 2$ line, transgene-activated homozygous males (CKTTA $+/-\mathrm{Tau}+/+$ ) were bred to nonactivated homozygous females (CKTTA-/-Tau+/+). Generation of rTg4510 mice, which utilizes an activator and responder system for transgene expression, has previously been described $^{2}$. Briefly, a pTRE-prnp-tau plasmid was used to generate $\operatorname{Tg} 4510$ responders, which harbor a $0 N 4 R$ human tau cDNA transgene regulated by a tetracycline response element (TRE). Activator mice harbor a tTA transgene under the control of the CaMKIIa promoter to drive expression specifically in forebrain excitatory neurons. Tau expression is activated in bigenic rTg4510 progeny of an activator-responder cross. For the rTg4510 line, responder Tg4510 were maintained on a FVB/N background while activator mice were maintained on a 129S6 background. To match the genetic background to that of rT2/T2 mice, we increased the amount of FVB/N and used these mice to compare expression levels in rTg4510 and rT2/T2 mice. To generate these mice, responder Tg4510 mice were maintained on a FVB/N background while activator mice were maintained on a mixed 129S6 and FVB/N background. Nontau-expressing transgenic littermates were used as controls. Both male and female mice were used, and were combined in statistical analyses after demonstrating the absence of significant gender effects $(P>0.05)$. All experiments with animals described in this study were approved by and conducted in full accordance with the American Association for the

Accreditation of Laboratory Animal Care and the Institutional Animal Care and Use Committee at the University of Minnesota.

qRT-PCR. mRNA expression levels of each Fgf14 variant were quantified relative to a reference gene, Hprt. All Fgf14 primers (Supplementary Table 1) were designed to span at least partially unique regions of each variant. Total cellular RNA was extracted from homogenized forebrain tissue using RNeasy Lipid Tissue Kit (Qiagen) according to the manufacturer's instructions. RNA samples were treated with DNaseI (New England Biolabs) to digest contaminating DNA, and subjected to cDNA synthesis using the iScript cDNA synthesis kit (Invitrogen) according to the manufacturer's instructions. PCR reactions were set up in a $20-\mu \mathrm{l}$ volume in $96-$ well plates, with 2-3 replicates per sample. SYBR Green PCR master mix (Roche) was used and reactions were run in the LightCycler ${ }^{\oplus} 480$ instrument (Roche) (Supplementary Table 2). A final melting curve confirmed that single amplicons 
were present for each variant and reference reactions, and a basic relative quantification was performed using the $\Delta \Delta \mathrm{C}_{\mathrm{T}^{-}}$-Method (LightCycler ${ }^{\circledR} 480$ Software release 1.5.0 SP3). All data were normalized to a positive-calibrator sample used in each experiment.

Protein extraction and phosphatase treatment. Total protein was extracted from mouse forebrain-hemisphere tissue in radioimmunoprecipitation assay (RIPA) buffer (50 mM tris- $\mathrm{HCl}, 150 \mathrm{mM} \mathrm{NaCl}, 1 \mathrm{mM}$ EDTA, 0.5\% Triton X-100, $1 \%$ sodium deoxycholate, $0.3 \%$ sodium dodecyl sulfate (SDS), $0.1 \mathrm{mM}$ phenylmethyl

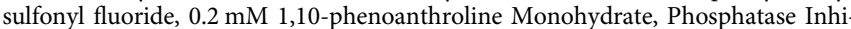
bitor Cocktail A (Sigma), Protease Inhibitor Cocktail (Sigma), Phosphatase Inhibitor Cocktail 2 (Sigma)). Homogenates were nutated and centrifuged at 15,700 $\times g$ for $90 \mathrm{~min}$ at $4{ }^{\circ} \mathrm{C}$ and the supernatant was collected ${ }^{31}$.

To obtain RIPA-insoluble, sarkosyl-insoluble fractions, a modified version of a previously published method was used ${ }^{31}$. RIPA-insoluble pellets were homogenized in $1 \%$ sarkosyl and incubated at room temperature for $30 \mathrm{~min}$ with constant shaking. Samples were centrifuged for $1 \mathrm{~h}$ at $100,000 \times g$ at $20^{\circ} \mathrm{C}$, and the supernatant and pellet were separated and diluted in $\mathrm{O}+$ buffer $(62.5 \mathrm{mM}$ tris- $\mathrm{HCl}, \mathrm{pH} 6.8 ; 10 \%$ glycerol; 5\% 2-mercaptoethanol; 2.3\% SDS; 1 mM EGTA; 1 mM EDTA; 1 mM PMSF; $1 \mathrm{mM} \mathrm{Na} \mathrm{VO}_{4} ; 1 \mathrm{mM} \mathrm{NaF} ; 10 \mu \mathrm{l} / \mathrm{ml}$ of protease inhibitor cocktail P8340; SigmaAldrich). Samples were boiled for $3 \mathrm{~min}$ and stored at $-20^{\circ} \mathrm{C}$

For treatment with calf intestinal alkaline phosphatase (CIP, New England Biolabs), samples were resuspended in $10 \mu \mathrm{l}$ CIP buffer $(100 \mathrm{mM} \mathrm{NaCl}, 50 \mathrm{mM}$ Tris-HCl, $10 \mathrm{mM} \mathrm{MgCl} 2,1 \mathrm{mM}$ dithiothreitol, EDTA-free protease inhibitor cocktail, pH 7.9) per $1 \mu \mathrm{g}$ protein. One unit CIP per $\mu$ g protein was added to the samples prior to incubation at $37^{\circ} \mathrm{C}$ for $30 \mathrm{~min}$. Samples were then concentrated using Amicon Ultra centrifugal filters (Millipore).

Western blot and analysis. For sarkosyl-insoluble fractions, total protein concentration was normalized according to pellet weights. Total protein concentrations for all other samples were determined by Pierce ${ }^{\text {ex }}$ Bicinchoninic Acid protein assay (Thermo Scientific). Equal amounts of protein for each sample were loaded and separated using SDS-PAGE on $10 \%, 10.5-14 \%$, or $10-20 \%$ tris- $\mathrm{HCl}$ gels (Bio Rad). Protein was transferred to nitrocellulose membranes (Bio Rad), which were blocked with $5 \%$ bovine serum albumin (Sigma) in $1 \times$ tris buffered saline buffer with Tween 20 (TBST) buffer (10 mM Tris-Base (Sigma), $0.2 \mathrm{M} \mathrm{NaCl}$ (Macron Chemicals), $0.1 \%$ Tween-20 (Sigma) pH 7.4). Protein was immunoblotted with Tau46 (Cell Signaling Technology \#4019, dilution 1:10,000), Tau13 (BioLegend \#MMS-520R, dilution 1:60,000), GAPDH(14C10) (Cell Signaling Technology \#2118, dilution 1:4000), GAPDH(GA1R) (Thermo Scientific \#MA5-15738, dilution 1:5000), AT8 (Thermo Scientific \#MN1020, dilution 1:1000), anti-human tau (Abcam \# ab74391, dilution 1:10,000), and BIII-tubulin (ProSci \#79-720, dilution 1:10,000) antibodies. Additional antibodies from Peter Davies for phosphoepitopes on tau included MC1 (dilution 1:800), CP13 (dilution 1:1,000), and PHF1 (dilution 1:1,500). To visualize antibody immunoreactivity using a LiCor imaging system and Image Studio software, IRDye-linked goat anti-mouse 800CW and goat anti-rabbit 680LT secondary antibodies were used (LI-COR Biosciences,dilution 1:100,000). Following LiCor image acquisition using Image Studio software (Odyssey), Amido black staining solution (Sigma-Aldrich) was used for total protein quantification according to manufacturer's instructions. Immunoreactivity and Amido black staining were quantified by densitometry using OptiQuant version 3 software, following guidelines for total protein quantification ${ }^{30}$.

Immunohistochemistry and analysis. Mouse brain hemispheres were immersionfixed in $10 \%$ formalin for $48 \mathrm{~h}$ before processing. Unstained sagittal TMA sections $(4 \mu \mathrm{m})$ were deparaffinized and rehydrated using standard methods. Bielschowsky silver staining was performed using standard techniques. For antigen retrieval, slides were incubated in $6.0 \mathrm{pH}$ buffer (Reveal Decloaking reagent, Biocare Medical, Concord, CA) in a steamer for $30 \mathrm{~min}$ at $95-98^{\circ} \mathrm{C}$, followed by a $20 \mathrm{~min}$ cool down period. Subsequent steps were automated using an immunohistochemical staining platform (Nemesis, Biocare). Endogenous peroxidase activity was quenched by slide immersion in 3\% hydrogen peroxide solution (Peroxidazed, Biocare) for 10 min followed by TBST rinse. A serum-free blocking solution (Rodent Block M, Biocare Medical, Concord, CA) was placed on sections for 20 min. Blocking solution was removed and slides were incubated in primary antibody diluted in $10 \%$ blocking solution/90\% TBST. Mouse monoclonal antibodies from Peter Davies were applied at the following dilutions: CP13 1:1000, MC-1 1:800 and PHF1 1:1500, mouse monoclonal PHF-Tau: clone AT8 (Thermo Scientific \#MN1020) $1: 1000$. Sections were incubated in primary antibody for $60 \mathrm{~min}$ at room temperature followed by TBST rinse and detection with biotinylated anti-mouse secondary (Vector Laboratories \#BP-9200, dilution 1:200) for 30 min followed by a TBST rinse. After the rinse, SA-HRP (Biolegend \#405210, RTU) was applied for $30 \mathrm{~min}$. All slides then proceeded with TBST rinse and detection with diaminobenzidine ${ }^{32}$ (Covance, Dedham, MA). Slides were incubated for $5 \mathrm{~min}$ followed by TBS rinse then counterstained with CAT Hematoxylin (Biocare, Concord, CA) for $5 \mathrm{~min}$. Slides were then dehydrated and coverslipped. Images were gathered using an Axioskop microscope (Zeiss, Germany) at 40× magnification and a PixeLINK microscope camera (PL-A623C) with PixeLINK Capture SE software version 2.2 (Firewire camera release 4, Copyright ${ }^{\odot} 2000-2006$ ). Adobe Photoshop CS2 version
9.0 was used to match the color of different images of the same histological stain. Semi-quantitative analysis of images was conducted using a " + " system ${ }^{1}$. A blinded observer gave scores for three sections per sample indicating severity of pathology using "-" for no positive labeling, "+" for occasional positive labeling, "++" for moderate positive labeling, " +++ " for prominent positive labeling. To summarize these results, the number of "+" signs was counted for each animal and region, and multiple linear regression analyses were conducted using $\mathrm{R}$ statistical programming language to test for differences between $\mathrm{rTg} 4510, \mathrm{rT} 2 / \mathrm{T} 2$, and rT2.

\section{Whole-genome sequencing and sequence analyses. Genomic DNA was} extracted from a non-activated Tg4510 mouse harboring the 0N4R human tau transgene using the DNeasy Blood \& Tissue kit (Qiagen) according to the manufacturer's instructions. Genomic DNA was analyzed by nanodrop and agarose gel to verify the quality (O.D. 260/280 ratio $>1.8$ ) and quantity (300 ng for library construction).

Sequencing was performed on Illumina HiSeq 2500 High-Output system using rapid SBS chemistry at the University of Minnesota Genomics Center, Minneapolis, MN. Following quality control, a TruSeq Nano DNA library was prepared from the genomic DNA sample and was sequenced on a single Illumina lane to generate $2 \times 125 \mathrm{bp}$ paired-end reads. The average insert length in the library was $350 \mathrm{bp}$.

All sequence analyses were conducted using the University of Minnesota's installation of the Galaxy web-based suite of software ${ }^{33}$. We isolated individual sequence reads that spanned the end of the transgene using the BLAT alignment tool $^{34}$ and mapped paired sequence reads to the transgene sequence using Bowtie $2^{35}$. We screened these data sets and identified unmapped reads in which the paired read mapped to the transgene sequence and then further analyzed these screened sets, in part using the Integrative Genomic Viewer ${ }^{36}$, to find the genomic insertion points and a single $5^{\prime}-5^{\prime}$ transgene fragment junction. Bowtie2 mapping of the sequence data to the Fgf14 genomic or Vipr2-Ptprn2 data (as appropriate) indicated that roughly half as many reads mapped to the unique sequences between the insertion points as mapped outside of this region, indicating that the $\operatorname{Tg} 4510$ mice have only a single copy of this portion of the Fgfl4 genomic sequence. To estimate transgene copy number, we used sets of closely linked SNPs to distinguish between the PrnP sequence in the transgene and that in the FVB genome $\mathrm{e}^{37}$, and found that the dataset contains more than 35 -fold more reads generated from transgene PrnP sequences than from the diploid mouse PrnP sequence on chromosome 2, indicating that more than 70 copies of transgene sequence are inserted at the Fgf 14 locus in the Tg4510 mice. Similar analyses were performed using polymorphisms in the CamKII genomic data to determine the copy number in the tTA transgene array. Sequences mapped to the reference Tg sequence were assemble into a consensus transgene sequence using SPADES $^{38}$.

PCR and sequencing. Selected regions of Tg4510 and rT2/T2 genomic DNA were amplified by PCR using Herculase II Fusion DNA polymerase (Agilent Technologies). For Tau transgene junctions in Tg4510 DNA, PCR and nested PCR reactions were run (Supplementary Tables 3-5). CaMKIIa-tTA transgene junctions were confirmed using rT2/T2 DNA (Supplementary Tables 6 and 7). Gel bands were isolated from a low melting point agarose gel (NuSieve GTG, Lonza) and DNA was purified following digestion with $\beta$-agarase (New England Biolabs). Gelpurified PCR products were sequenced with classical Sanger sequencing at the University of Minnesota Genomics Center, Minneapolis, MN.

Mouse cell culture and metaphase slide preparation. Spleen from a Tg4510 mouse (28th generation backcross onto FVB genetic background) was minced into a single-cell suspension and cultured for $24-28 \mathrm{~h}$ with $5 \mu \mathrm{g} / \mathrm{ml}$ Concavalin A (mouse T-cell mitogen). Cultures were exposed to colcemid (Irvine Scientific) for $15 \mathrm{~h}$ overnight followed by harvest using standard cytogenetic protocols. Metaphase spread slides were prepared from methanol-acetic acid fixed cell pellets and FISH was performed the following day.

Fluorescent in situ hybridization. DNA probes derived from the tau transgene sequence were labeled by nick translation reaction (Nick Translation Kit-Abbott Molecular) using Orange 552 dUTP (Enzo Life Science), ethanol precipitated and resuspended in hybridization buffer. The probe/hybridization buffer mix and slide were denatured, probe was applied to the metaphase slide, and slide was hybridized for $24 \mathrm{~h}$ at $37^{\circ} \mathrm{C}$ in a humidified chamber. After hybridization, the FISH slides were washed in a $2 \times$ SSC solution and counterstained with DAPI stain to enable chromosome identification by G-band patterning. Fluorescent signals were visualized on an Olympus BX61 microscope workstation (Applied Spectral Imaging, Vista, CA) with DAPI and Texas Red filter sets. FISH images were captured using an interferometer-based CCD cooled camera (ASI) and FISHView ASI software.

Behavioral experiments. For nesting experiments, animals were not disturbed for at least $24 \mathrm{~h}$ prior to testing. Each animal was placed in its own clean cage with a new nestlet placed in the center of the cage in the morning. Nests were photographed and scored 2, 6, and $24 \mathrm{~h}$ after the start of the test. Scores of $0-7$ were given as follows ${ }^{9}$ : $0-$ nestlet untouched, $1-<10 \%$ of nestlet was shredded, $2-10-50 \%$ of nestlet shredded but no shape to nest (flat), 3-10-50\% of nestlet shredded and 
there is shape to nest, $4-50-90 \%$ of nestlet shredded but no shape to nest (flat), $5-50-90 \%$ of nestlet shredded and there is shape to nest, $6 \rightarrow 90 \%$ of nestlet shredded but no shape to nest (flat), $7 \longrightarrow>90 \%$ of nestlet shredded and nest had walls that were at least as tall as the mouse on $50 \%$ of the sides. Reported scores are the average of two individual blinded scorers.

Open field testing was done two days after the conclusion of nesting experiments. Animals were not disturbed for at least $24 \mathrm{~h}$ prior to testing and were placed in the testing room for $30 \mathrm{~min}$ prior to testing. The Open field setup was a plastic tub with opaque white walls, measuring 15 in wide by 18.5 in long by 12 in high. The arena floor was covered with new, prescented cage bedding. Each mouse was released in the center of the arena and allowed to freely explore for $10 \mathrm{~min}$. All trials were monitored using a computerized tracking system (Noldus Ethovision XT 10.0; Noldus Information Technology). All animals were tested in the morning to avoid activity differences from time of day.

Statistical analysis. Analyses were conducted using GraphPad Prism version 6.00 software (GraphPad Software) and R statistical programming language. Gender differences were detected only in nest-building behavioral assays and analyses were conducted accordingly. In all cases, $p<0.05$ was considered to be statistically significant.

Availability of unique biological materials. We will not be able to distribute mouse ES cells, since they will not tolerate repeated passaging, but all other materials and mouse lines will be available for distribution when requested.

Reporting summary. Further information on research design is available in the Nature Research Reporting Summary linked to this article.

\section{Data availability}

Sequence data that support the findings of this study (e.g., Figs. 3 and 5, and Supplementary Figs. 2 and 3) have been deposited in GenBank with the primary accession codes MF989990 (Tg4510 transgene), MF989991 (head-to-tail transgene junction), MF989992 (head-to-head transgene junction), MF989993 (transgene arrayFgf14 promoter region junction), and MF989994 (transgene array-Fgf14 intron junction). Accession numbers for tTA-TgINDEL sequences are MK674482 (CamKII-tTA transgene_monomer), MK674483 (CamKII-tTA Tg-Vector-Tg junction), MK674484 (CamKII-tTA_Tg-Ptrn2_junction), MK674485 (Vipr2-CamKII-tTA Tg junction), MK674486 (CamKII-tTA Tg head-to-tail junction Type 1), MK674487 (CamKII-tTA Tg head-to-tail junction Type 2), MK674488 (CamKII-tTA Tg head-to-tail junction Type 3). The source data underlying Figs. 1a-d, 2a, b, 4a-e, 6b, and 7a-e and Supplementary Figs. 9b, 10b, 11, and 12a, b are provided as a Source Data file.

Received: 10 July 2018 Accepted: 10 May 2019

Published online: 06 June 2019

\section{References}

1. Ramsden, M. et al. Age-dependent neurofibrillary tangle formation, neuron loss, and memory impairment in a mouse model of human tauopathy (P301L). J. Neurosci. 25, 10637-10647 (2005)

2. Santacruz, K. et al. Tau suppression in a neurodegenerative mouse model improves memory function. Science 309, 476-481 (2005).

3. Gotz, J., Chen, F., Barmettler, R. \& Nitsch, R. M. Tau filament formation in transgenic mice expressing P301L tau. J. Biol. Chem. 276, 529-534 (2001). M006531200

4. Lewis, J. et al. Neurofibrillary tangles, amyotrophy and progressive motor disturbance in mice expressing mutant (P301L) tau protein. Nat. Genet 25, 402-405 (2000).

5. Mayford, M. et al. Control of memory formation through regulated expression of a CaMKII transgene. Science 274, 1678-1683 (1996).

6. Hoover, B. R. et al. Tau mislocalization to dendritic spines mediates synaptic dysfunction independently of neurodegeneration. Neuron 68, 1067-1081 (2010).

7. Beard, C., Hochedlinger, K., Plath, K., Wutz, A. \& Jaenisch, R. Efficient method to generate single-copy transgenic mice by site-specific integration in embryonic stem cells. Genesis 44, 23-28 (2006).

8. Pardo, L. M. et al. Regional differences in gene expression and promoter usage in aged human brains. Neurobiol. Aging 34, 1825-1836 (2013).

9. Maeda, S. et al. Expression of A152T human tau causes age-dependent neuronal dysfunction and loss in transgenic mice. EMBO Rep. 17, 530-551 (2016).

10. Shineman, D. W. et al. Accelerating drug discovery for Alzheimer's disease: best practices for preclinical animal studies. Alzheimers Res. Ther. 3 , 28 (2011).
11. Yue, F. et al. A comparative encyclopedia of DNA elements in the mouse genome. Nature 515, 355-364 (2014).

12. Smith, E. D. et al. More than blood, a novel gene required for mammalian postimplantation development. Mol. Cell Biol. 24, 1168-1173 (2004).

13. Han, H. J. et al. Strain background influences neurotoxicity and behavioral abnormalities in mice expressing the tetracycline transactivator. J. Neurosci. 32, 10574-10586 (2012).

14. Helboe, L., Egebjerg, J., Barkholt, P. \& Volbracht, C. Early depletion of CA1 neurons and late neurodegeneration in a mouse tauopathy model. Brain Res. 1665, 22-35 (2017).

15. Bejar, R., Yasuda, R., Krugers, H., Hood, K. \& Mayford, M. Transgenic calmodulin-dependent protein kinase II activation: dose-dependent effects on synaptic plasticity, learning, and memory. J. Neurosci. 22, 5719-5726 (2002).

16. Di, Re,J., Wadsworth, P. A. \& Laezza, F. Intracellular fibroblast growth factor 14: emerging risk factor for brain disorders. Front. Cell. Neurosci. 11, 103 (2017).

17. Wang, Q., McEwen, D. G. \& Ornitz, D. M. Subcellular and developmental expression of alternatively spliced forms of fibroblast growth factor 14. Mech. Dev. 90, 283-287 (2000).

18. Laezza, F. et al. FGF14 N-terminal splice variants differentially modulate Nav1.2 and Nav1.6-encoded sodium channels. Mol. Cell Neurosci. 42, 90-101 (2009).

19. Pablo, J. L., Wang, C., Presby, M. M. \& Pitt, G. S. Polarized localization of voltage-gated $\mathrm{Na}+$ channels is regulated by concerted FGF13 and FGF14 action. Proc. Natl Acad. Sci. USA 113, E2665-E2674 (2016).

20. Smallwood, P. M. et al. Fibroblast growth factor (FGF) homologous factors: new members of the FGF family implicated in nervous system development. Proc. Natl Acad. Sci. USA 93, 9850-9857 (1996).

21. Xiao, M. et al. Impaired hippocampal synaptic transmission and plasticity in mice lacking fibroblast growth factor 14. Mol. Cell Neurosci. 34, 366-377 (2007).

22. Alshammari, T. K. et al. Genetic deletion of fibroblast growth factor 14 recapitulates phenotypic alterations underlying cognitive impairment associated with schizophrenia. Transl. Psychiatry 6, e806 (2016).

23. Alshammari, M. A., Alshammari, T. K., Nenov, M. N., Scala, F. \& Laezza, F. Fibroblast growth factor 14 modulates the neurogenesis of granule neurons in the adult dentate gyrus. Mol. Neurobiol. 53, 7254-7270 (2016).

24. Lou, J. Y. et al. Fibroblast growth factor 14 is an intracellular modulator of voltage-gated sodium channels. J. Physiol. 569, 179-193 (2005).

25. Misceo, D. et al. SCA 27 caused by a chromosome translocation: further delineation of the phenotype. Neurogenetics 10, 371-374 (2009).

26. Cook, C. et al. Tau deposition drives neuropathological, inflammatory and behavioral abnormalities independently of neuronal loss in a novel mouse model. Hum. Mol. Genet. 24, 6198-6212 (2015).

27. Andorfer, C. et al. Cell-cycle reentry and cell death in transgenic mice expressing nonmutant human tau isoforms. J. Neurosci. 25, 5446-5454 (2005)

28. Saito, T. et al. Single App knock-in mouse models of Alzheimer's disease. Nat. Neurosci. 17, 661-663 (2014).

29. Sasaguri, H. et al. APP mouse models for Alzheimer's disease preclinical studies. EMBO J. 36, 2473-2487 (2017).

30. Aldridge, G. M., Podrebarac, D. M., Greenough, W. T. \& Weiler, I. J. The use of total protein stains as loading controls: an alternative to high-abundance single-protein controls in semi-quantitative immunoblotting. J. Neurosci. Methods 172, 250-254 (2008).

31. Planel, E. et al. Acceleration and persistence of neurofibrillary pathology in a mouse model of tauopathy following anesthesia. FASEB J. 23, 2595-2604 (2009).

32. Dabir, D. V. et al. Impaired glutamate transport in a mouse model of tau pathology in astrocytes. J. Neurosci. 26, 644-654 (2006).

33. Afgan, E. et al. The Galaxy platform for accessible, reproducible and collaborative biomedical analyses: 2016 update. Nucleic Acids Res. 44, W3-W10 (2016).

34. Kent, W. J. BLAT-the BLAST-like alignment tool. Genome Res. 12, 656-664 (2002). Article published online before March 2002.

35. Langmead, B. \& Salzberg, S. L. Fast gapped-read alignment with Bowtie 2. Nat Methods 9, 357-359 (2012).

36. Thorvaldsdottir, H., Robinson, J. T. \& Mesirov, J. P. Integrative Genomics Viewer (IGV): high-performance genomics data visualization and exploration. Brief. Bioinform 14, 178-192 (2013).

37. Westaway, D. et al. Structure and polymorphism of the mouse prion protein gene. Proc. Natl Acad. Sci. USA 91, 6418-6422 (1994).

38. Bankevich, A. et al. SPAdes: a new genome assembly algorithm and its applications to single-cell sequencing. J. Comput. Biol. 19, 455-477 (2012).

\section{Acknowledgements}

We thank the University of Minnesota Genomics Center (UMGC), Daryl Gohl in particular, for advising us in experimental design and for conducting the whole-genome 
sequencing experiments. We acknowledge Kevin Silverstein at the Minnesota Supercomputing Institute (MSI), whose suggestions for computational approaches aided our identification of the insertion location of the transgene sequence. In addition, MSI's computational research infrastructure was used for this task. We thank Kailee Leinonen, Elizabeth Steuer, and Eric Huang for their assistance with mouse breeding, genotyping, and behavioral tests. Cytogenetic analyses were performed in the Cytogenomics Shared Resource at the University of Minnesota with support from the comprehensive Masonic Cancer Center NIH Grant \#P30 CA077598. This research received histology assistance from the University of Minnesota's Biorepository and Laboratory Services program and was supported by the National Institutes of Health's National Center for Advancing Translational Sciences, grant UL1TR002494. The content is solely the responsibility of the authors and does not necessarily represent the official views of the National Institutes of Health's National Center for Advancing Translational Sciences. Partial support for EF was provided by the Office of the Director, National Institutes of Health (NIH) under award number K01-OD019912. This work was supported in part by a Zenith Award from the Alzheimer's Association to M.K. and K.A.

\section{Author contributions}

M.K. and K.A. directed the study and made intellectual contributions to the experimental design and discussion. K.B. and M.K developed the T2 mouse line, J.G. bred and performed all of the characterization of the rT2/T2 and control lines discussed, other than tau histopathology, which was conducted primarily by C.F. Behavior experiments were performed by L.K. C.H. assisted with statistical analyses of tau histopathology. Genomic sequence analysis was performed by M.K. with assistance from E.F., and the paper was written primarily by M.K. and J.G. with input from K.A.

\section{Additional information}

Supplementary Information accompanies this paper at https://doi.org/10.1038/s41467019-10428-1.
Competing interests: The University of Minnesota has certain ownership rights in the rTg4510 mouse and has received royalty income pursuant to a licensing agreement with the Mayo Medical Foundation, which holds the patent on $\mathrm{rTg} 4510$ mice; a portion of that royalty income has been distributed to K.A. No royalty income has been received by K.A. since May 2016. The remaining authors declare no competing interests.

Reprints and permission information is available online at http://npg.nature.com/ reprintsandpermissions/

Journal peer review information: Nature Communications thanks the anonymous reviewers for their contribution to the peer review of this work.

Publisher's note: Springer Nature remains neutral with regard to jurisdictional claims in published maps and institutional affiliations.

cC (i) Open Access This article is licensed under a Creative Commons Attribution 4.0 International License, which permits use, sharing, adaptation, distribution and reproduction in any medium or format, as long as you give appropriate credit to the original author(s) and the source, provide a link to the Creative Commons license, and indicate if changes were made. The images or other third party material in this article are included in the article's Creative Commons license, unless indicated otherwise in a credit line to the material. If material is not included in the article's Creative Commons license and your intended use is not permitted by statutory regulation or exceeds the permitted use, you will need to obtain permission directly from the copyright holder. To view a copy of this license, visit http://creativecommons.org/ licenses/by/4.0/.

(c) The Author(s) 2019 